\title{
Dermoscopy of pigmented oral lesions
}

\author{
Malgorzata Olszewska ${ }^{1}$, Agnieszka Banka ${ }^{1}$, Renata Gorska², Olga Warszawik ${ }^{1}$
}

1. Dept. Dermatology, Warsaw Medical University, Poland

2. Dept. Periodontology and Oral Diseases, Warsaw Medical University, Poland

\section{Corresponding author:}

Malgorzata Olszewska, MD, PhD

Dept. Dermatology

Warsaw Medical University

Koszykowa 82a

02-008 Warsaw, Poland

E-mail: malgorzataolszewska@yahoo.com

\section{Key words:}

dermoscopy, mouth, oral manifestation, mucous membrane, lip, melanoma, tongue

\section{Abstract}

Dermoscopy is a noninvasive diagnostic technique that permits the visualization of morphologic features that are not visible to the naked eye. It is currently widely used for examination of pigmented skin lesions and early detection of cutaneous melanoma. Dermoscopy of mucous membranes is significantly less investigated and less popular among clinicians.

This article reviews current knowledge about characteristic dermoscopy features of pigmented lesions of the oral mucous membranes and lips. It has to emphasized that a major advantage of dermoscopy is the capability to exclude melanoma and avoid unnecessary excisional biopsies and extensive surgery, often resulting in significant disfigurement of face and oral cavity.

It has to be pointed out that there is a necessity to intensify research, which would result in clear-cut dermoscopy criteria for mucosal melanoma and the need for constructing thin, flexible dermoscopes suitable for investigation of oral mucous membranes.
Dermoscopy is a noninvasive diagnostic technique that permits the visualization of morphologic features that are not visible to the naked eye. It is currently widely used for examination of pigmented skin lesions and early detection of cutaneous melanoma. ${ }^{1,2}$

Despite increasing popularity in examination of skin lesion, the methods remains unpopular in investigating pigmented lesions oral mucous membranes and lips.

Pigmented mucosal lesions represent a group of rare entities differing in etiopathogenesis, histology, dermoscopy and clinical course. Solitary pigmented lesions of melanocytic origin are uncommon in the oral mucosa. These lesions include the oral and labial melanotic macules, oral melanocytic nevi, oral melanoacanthomas and oral melanomas. ${ }^{3}$ As shown by Buchner et al 4 oral and labial melanotic macules are the most common melanocytic lesions. The relative frequency of oral melanoma accounts for $0.6 \%$ of the entire melanocytic group of oral pigmented lesions (Table 1). Despite low frequency, early detection of melanoma is the main aim of dermoscopic examinations of the oral mucous membranes and the lip. An additional benefit of dermoscopy is exclusion of melanoma of the oral mucous membranes or the lip in doubtful cases, what may save patients the discomfort of extensive and unnecessary surgery.

In this paper, we review current knowledge about dermoscopy of pigmented lesions localized on the mucous membranes of the oral cavity and the semi-mucosa of lips.

Table 1

Relative frequency of oral solitary melanocytic lesions. ${ }^{4}$

\begin{tabular}{|l|l|}
\hline Type of lesion & Frequency \\
\hline Melanotic macule & $86,1 \%$ \\
Melanocytic nevi & $11,8 \%$ \\
Melanoacanthoma & $0,9 \%$ \\
Oral melanoma & $0,6 \%$ \\
Other & $0,6 \%$ \\
\hline
\end{tabular}




\section{Melanocytic nevi}

Intraoral and labial melanocytic nevi are uncommon and occur with an estimated frequency of about 1 in 1000 people. ${ }^{5}$ The clinical significance of these lesions lies in their possible predilection to transform into melanoma and in difficult differential diagnosis between these two entities. In about $30 \%$ of all oral melanomas pigmented lesion of unknown origin was reported to precede melanoma. ${ }^{6,7}$ It may be hypothesized, that a majority of these preceding lesions were melanocytic nevi.

Corresponding to classification on cutaneous nevi, oral nevi can be intramucosal, junctional, compound, or blue nevi. A nevus with nevus cells located in the basal layer of epithelium (epithelial-connective tissue junction) is referred to as a junctional nevus. When nevus cells are located in the epithelium and in the lamina propria it is a compound nevus. When nevus cells are located in the lamina propria, it is an intramucosal nevus. Intramucosal nevi are most common and represent about $55-64 \%$ of all nevi located in the oral cavity. ${ }^{8,9}$

Oral melanocytic nevi may occur at any age. Usually they appear in young adults, at the age of 20-40. They are more common in women than in men. ${ }^{10}$

Clinically, nevi are well circumscribed, round or oval, usually brown, sometimes grey or black. 10 Nevi are often slightly elevated, what is an important feature in differentiating nevi from amalgam tattoos or melanocytic macules, which are flat.

Up to $15 \%$ of oral nevi can be non-pigmented. This is usually the case in intramucosal nevi. ${ }^{11}$ Non-pigmented nevi present a particular diagnostic challenge.

Oral nevi are usually small. About $75 \%$ of them are less than $0,6 \mathrm{~mm}$ in diameter and $50 \%$ are $0,1-0,3$ in diameter. ${ }^{12}$ Lesions bigger than $1,3 \mathrm{~cm}$ are rare. ${ }^{12}$

Dermoscopic examination reveals well circumscribed, symmetric lesions, homogeneous in structure, often with broadened or prominent pigment network and streaks. ${ }^{13}$

Dermoscopic monitoring of these lesions is of special significance, to ensure early diagnosis in case of malignant transformation. Most authors suggest, that any pigmented lesion within the oral mucous membranes should be excised to exclude possible misdiagnosis of melanoma. Only a minority of authors ${ }^{14}$ express the opinion, that presence of intraoral melanocytic nevi is not associated with increased risk of melanoma.

A distinct form of a intraolral or labial nevus is the intraoral blue (Jadassohn-Tieche) nevus. Blue nevi make up a significant promotion of all reported intraoral melanocytic nevi. According to some authors 11 , blue nevi in oral mucous membranes are overdiagnosed, because of their similarity to other oral lesions.

Our limited experience shows that dermoscopy of a mucosal blue nevus exhibits a homogeneous bluish lesion, accompanied by blue-whitish veil-like structures. As in other mucosal and labial lesions, a blue nevus is surrounded by multiple regularly structured blood vessels. Reports of further cases of various types of mucosal blue nevi are needed to reveal the possible range of dermoscopy variants of these lesions. It requires dermoscopic differential diagnosis with other pigmented lesions amalgam tattoos.

\section{Oral mucosal melanoma}

Intraoral mucosal melanoma is a very rare neoplasm. It is estimated to represent $1-2 \%$ of all oral malignancies and accounts for about $0,1-8 \%$ of all melanomas. $6,7,15$ Mean age of onset is 55-60,6,7 however there have been reports of onset in prepubertal children. ${ }^{16}$

Unlike cutaneous melanoma, oral melanoma is very frequent in Japan, Uganda and India. Among the Japanese oral melanoma accounts for $7.5 \%$ of all melanomas, versus less then $1 \%$ for Caucasians. It is twice more common in man than in woman. $17,18,19$

The predominate site of intraoral melanoma is the hard plate accounting for two thirds of all oral melanomas. Other sites include the maxillary alveolus, mandible, tongue and buccal mucosa.20,21

In about one third of all oral melanomas the tumor is preceded by preexisting pigmented lesion, most probably a nevus. ${ }^{7}$ In all remaining cases the anamnesis is negative, indicating de novo development of the tumor.

Clinically, oral mucosal melanoma most often presents as a solitary, brown to black macule or sometimes nodule, with asymmetric and irregular borders. It is estimated, that $5-35 \%$ of all oral melanomas are amelanotic, 19,22 which makes the diagnosis difficult. Typical features include rapid vertical growth and ulcer development within the lesion. 19,22

Melanoma of the oral cavity may occur with or without a radial growth phase and it may clinically classified as

\section{Pigmented nodular}

2. Nonpigmented nodular

3. Pigmented macular

4. Pigmented mixed

\section{Nonpigmented mixed type. ${ }^{7}$}

The prognosis of oral mucosal melanoma is usually poor, as a result of rapid growth and delayed diagnosis. The main reason for delayed diagnosis and treatment is uncharacteristic clinical appearance. Lack of features characteristic of melanoma in early phases of disease causes hesitation to performexcisional biopsies with removal of face or oral structures. By performing such surgery we may produce significant disfigurement with possible impairment in ability to chew, swallow or speak, while having only a low level of certainty in diagnosis of a malignant tumor.

Dermoscopy has emerged as an effective adjunctive tool in the in vivo examination of pigmented skin lesions and early diagnosis of cutaneous malignant melanoma. Experience in this regard indicates that the value of dermoscopy in early diagnosis of mucosal and labial melanoma may be considerable. However only limited data 


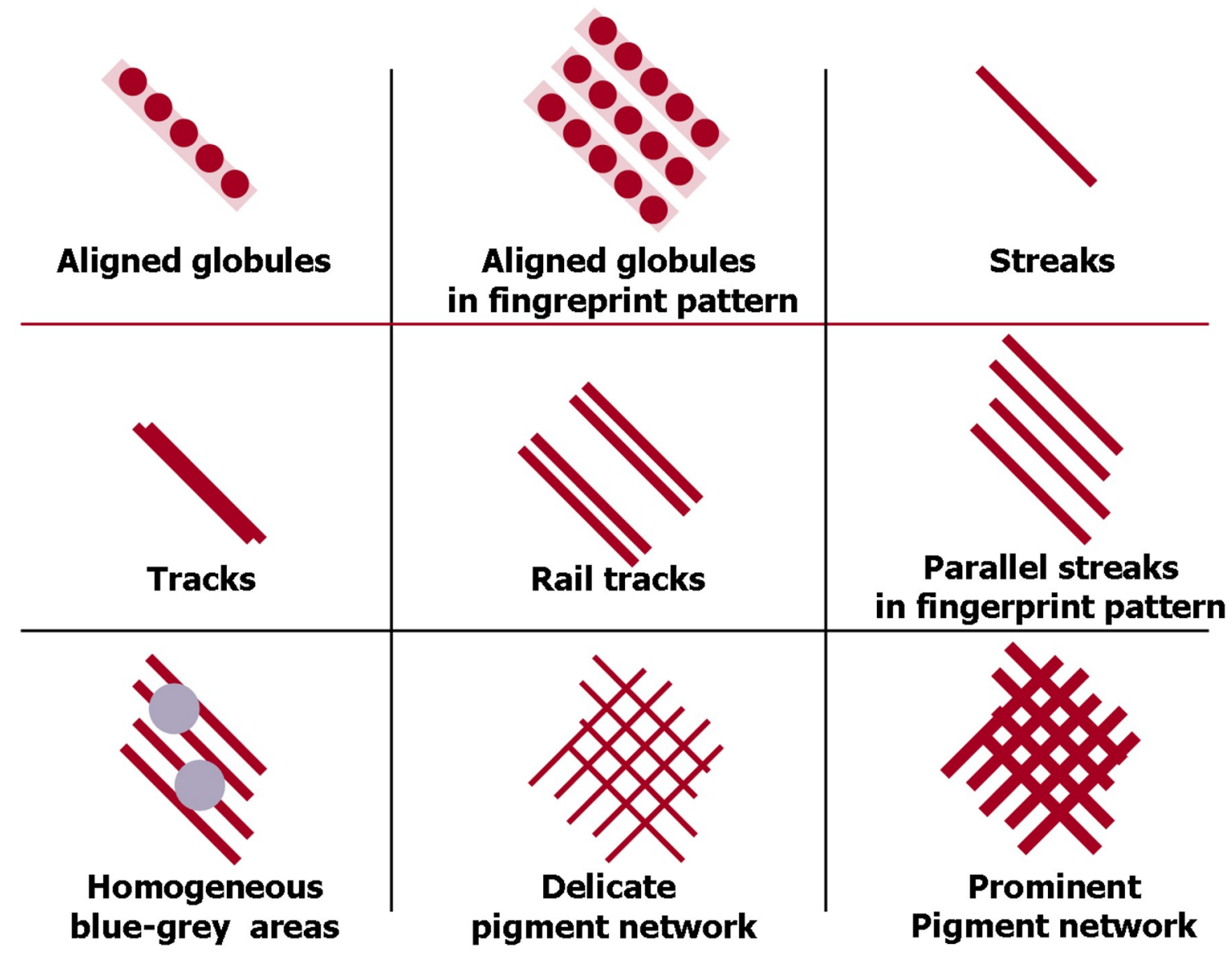

Figure 1

Patterns observed in dermoscopy of mucous membranes.

about dermoscopic features of mucosal lesions are available.9,13,23,24,25 Puig and Malvehi13 developed a simplified graph, which illustrates various patterns observed in dermoscopy of mucous membranes and assists in differential diagnosis of pigmented oral lesions (Fig. 1). However no large-scale study was performed to define dermoscopy criteria for oral and labial melanoma. An ongoing study on dermoscopy of pigmented and non-pigmented mucosal lesions, coordinated by professor Andreas Blum (Germany) promises new data in this regard.

Currently, data obtained on the basis of single labial and oral melanoma cases are available.

Matsushita et al 23 reported a case of labial melanoma, which showed in dermoscopy a picture of irregular diffuse pigmentation with a pseudo-network, accompanied by regression structures and a blue-whitish veil. Our experience confirms, that this is the most typical pattern, not only within the semi-mucosa but also in the mucous membranes (Fig. 2). This pattern is clearly different compared to amalgam tattoos (Fig. 3), despite frequent similarity upon macroscopic evaluation.

According to Puig and Malvehy ${ }^{13}$, the dermoscopic appearance of acral lentiginous melanoma of mucous membranes may be different in "in situ" and in invasive melanoma. Common features include heterogeneity of

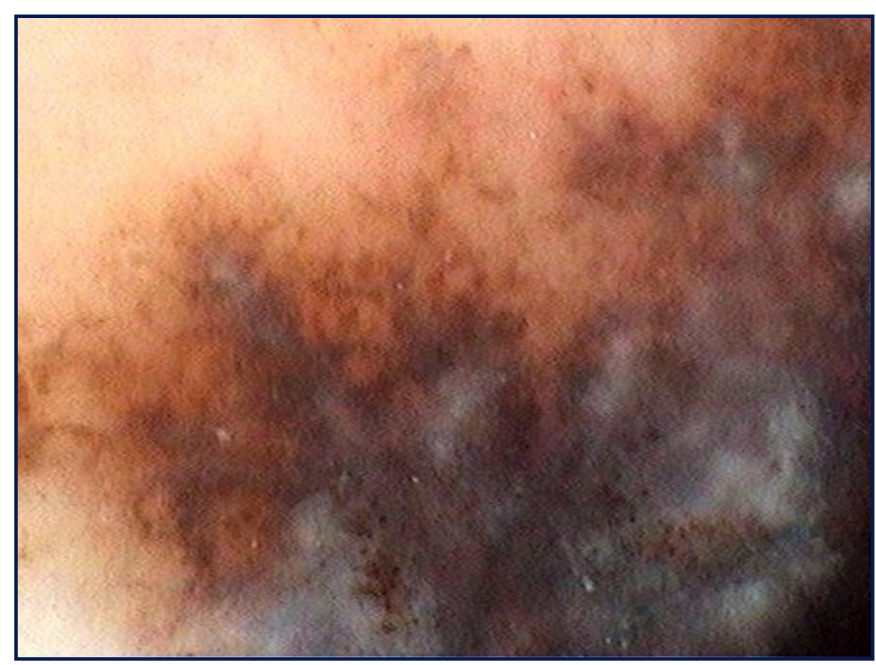

\section{Figure 2}

Dermoscopy of mucous melanoma. Irregular diffuse pigmentation with a pseudo-network, accompanied by regression structures and a blue-whitish veil.

both color and structures and abrupt cut-off of the pigment pattern at the periphery of the lesion. In "in situ" melanoma some areas show a globular aligned pattern that can reassemble an idiopatic melanosis whereas other areas show large blue-grey structures or irregularly 


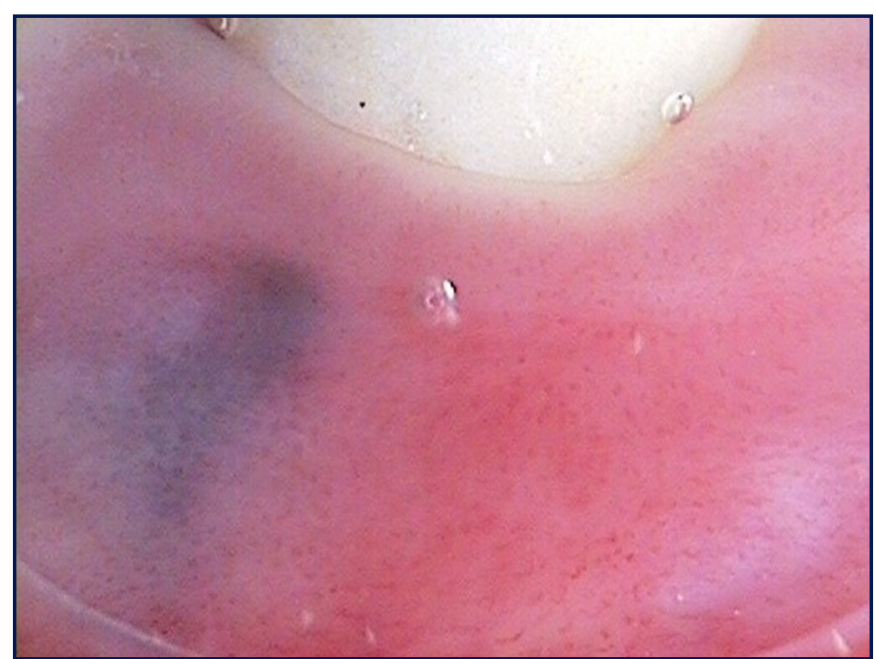

Figure 3

Dermoscopy of an amalgam tattoo. A structureless pattern.

distributed dots and globules. In invasive melanoma, blue-whitish veil, ulceration and atypical vascular pattern can be present. ${ }^{23}$

In a case shown by Stolz et a/26 dermoscopy showed an irregular serrated boarder, asymmetrically pigmented rete ridges and variably-sized granules.

Further studies are needed to develop dermoscopy criteria for melanoma of oral mucous membranes and the lip and to clarify in what extend dermoscopy of oral melanoma shows similarity to the corresponding image in genital mucous membranes.

However low frequency of oral melanoma, restricted familiarity of oral medicine specialists with dermoscopy and, most importantly, lack of dermoscopic equipment, which would allow visualization of lesions located intraorally is creating inhibits progress in this regard. In our opinion creating suitable, flexible dermoscopes with disposable tips is an important task for developers of dermoscopic devices.

\section{Melanotic macules (mucosal mela- nosis)}

Melanotic macule is the most common pigmented lesion located in the oral cavity. ${ }^{4}$ Lesions located on the lip are termed lip melanotic macule, whereas the those located intra-orally are termed oral melanotic macule. ${ }^{11}$

Melanotic macules are benign lesions and have a tendency to spontaneous regression. Aetiopathogenesis of these lesions is still unknown, however, local trauma and chronic inflammation are considered as a possible inducing factor of melanotic macules.27,28 Histologically, these lesions are characterized by the increased number of melanin in basal keratinocytes with normal number of melanocytes. ${ }^{27,28}$

Clinically, melanotic macule is a brown to black, small (usually less than $1 \mathrm{~cm}$ in diameter), flat, well-circumscribed, asymptomatic lesion. ${ }^{29}$ The vermillion border is the most common site $(31 \%)$. About $91 \%$ of these lesion are located on the lower lip, whereas only $6 \%$ on the upper lip. ${ }^{28,29}$ Other common locations are gingiva, palate and buccal mucosa. The glans penis and female genitalia may be other locations of melanocytic macules.9,13,26

A melanotic macule is usually a solitary lesion, but occasionally more than one lesion can be observed. They are more common in women than in men and usually occur in fourth or fifth life decade. ${ }^{28,29}$

Multiple labial and oral melanotic macules associated with linear streaks of nail pigmentation can be a symptom of Laugier-Hunziker syndrome. ${ }^{30}$

Cases of multiple melanocytic macules have been reported in immunosuppressed patients. ${ }^{31}$

It is not fully clarified whether dermoscopy of melanocytic macules varies depending on location between oral mucous membranes, lip, vulva and penile mucosa. In most reports melanocytic macules in these locations were evaluated together.

In a first description of Carli et a/32 it was emphasized, that labial, melancyotic macules appear in dermoscopy as regular and homogeneous, diffuse pigmentation differing from melanoma by lack of brown globules, radial streaming and pseudopods. In another case of labial melanocytic macule published by the same group of authors dermoscopy showed a homogeneous pigmentation, regularly distributed and gradually fading toward the periphery.

Mannone et a/33 distinguished 3 types of melanocytic macules, based on dermoscopic features of these lesions: (1) structureless, (2) parallel and (3) reticular type. A "structureless" pattern is predominantly found in vulvar melanosis, with a blue hue, associated with the presence of melanophages in the upper dermis, present in the majority of these lesions. A "parallel pattern", is often found in clinically typical melanotic macules of the lips and penis. A "reticular-like" pattern is associated with clinically equivocal melanosis occurring at peculiar sites such as the areola or, rarely, on the lip. ${ }^{33}$

Puig and Malvehi 33 emphasize that in bigger magnifications brown or black globules in a papillary / fingerprint pattern can be observed. In some cases or when observed in lower magnification narrow parallel lines or broadened track-like pigmentation can be observed. Authors also suggest, that this pattern of pigmented globules can be observed in mucosal melanoma, however, in cases of melanoma it is always associated with other dermoscopic features characteristic for melanoma. ${ }^{33}$

Stolz et a/26 indicated that benign pigmented lesions of the mucous membranes, forming a fingerprint pattern with narrow parallel lines and broader track-like pigmentation interrupted by dots and globules. According to our experience this is by far the most common pattern seen in melanocytic macules of the lips and oral mucous membranes. In many cases we observed a mixed pattern with more or less prominent, structureless brown areas (Fig. 4). This would correspond to a mixed parallel-structureless pattern according to the classification by Mannone et al. .33 


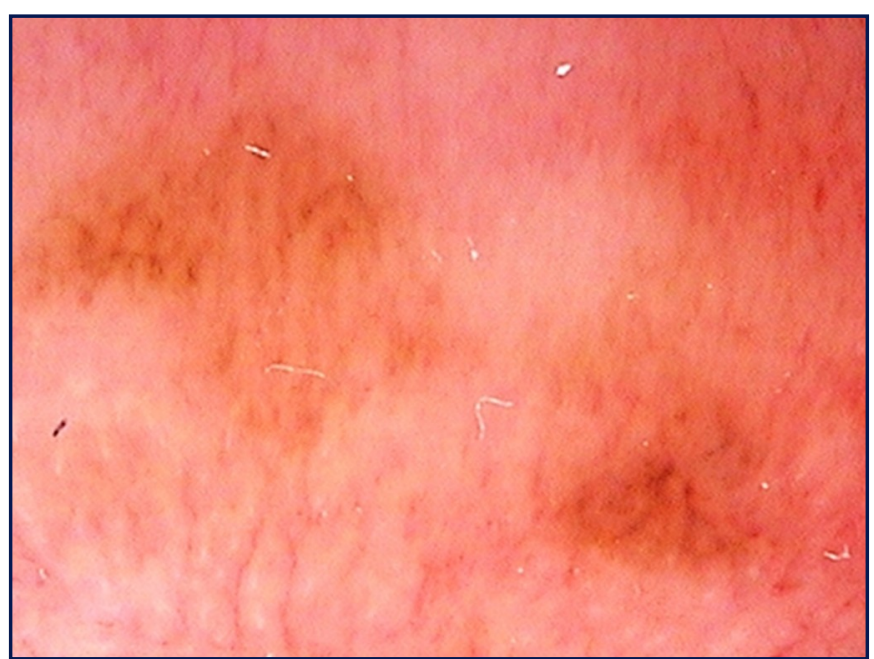

Figure 4

Dermoscopy of a melanocytic macule of the lip. A dominating fingerprint pattern with narrow parallel lines and broader track-like pigmentation.

\section{Oral Melanoacanthoma}

Melanoacanthoma is a term that has been introduced to describe a pigmented, benign proliferation of both keratinocytes and dendritic melanocytes. Oral melanoacanthoma is a rare pigmented mucosal lesion that is considered the counterpart of cutaneous melanoacanthoma. Oral melanoacanthoma is a rare pigmented lesion, which is most commonly located on the buccal mucosa. Lesions are usually solitary, but multiple melanoacanthomas were also reported. $34,35,36$ Melanoacanthoma is considered to be caused by trauma or irritation. Clinically, these lesions present as asymptomatic, slightly elevated, well circumscribed plaques with irregular surface. Lesions are usually brown, but sometimes intensely hyperpigmentatied or light brown. Melanoacanthoma can enlarge in a short period of time, which may be suggestive of malignancy. Histologic examination reveals presence of dendritic melanocytes throughout all layers of the epithelium, acanthosis, elongation and widening of rete ridges. ${ }^{34,35,36}$

Dermoscopic pattern of oral melanoacanthoma has not been established yet. In a case of cutaneous melanoacanthoma it has been reported that the lesion shows dermoscopically a stereotypical starburst pattern characterized by pigmented streaks symmetrically distributed at the periphery, which is considered to be characteristic for Spitz nevus. ${ }^{37}$

\section{Lentigo simplex}

Lentigo simplex typically occurs in elderly people. In some cases chronic exposition to UV is considered to be etiologic factor. Usually lesions are brown to black in colour, flat, well circumscribed, 0,2-2 cm in diameter. Most common are solitary lesions, typically located on lips, palate and gingiva. Histologic examination shows an increased level of melanin in basal cells and slight hyperplasia of melanocytes and of epithelium cells. ${ }^{8}$

Dermoscopic features of solar lentigo are aligned pigmented globules, linear tracks or streaks, occasionally delicate pigment network. ${ }^{13}$

\section{General issues}

Taking into consideration the risk of melanoma, every pigmented lesion located on mucous membranes of oral cavity or labial semimucosa should be diagnosed carefully. Most clinicians prefer to excise any pigmented oral lesion within oral mucous membranes and lip to exclude melanoma, although the disease is relatively infrequent.

Dermoscopy is a noninvasive diagnostic technique, which is currently widely used for examination of pigmented skin lesions and early detection of cutaneous melanoma. It has to be stressed, that dermoscopic features of lesions located on the mucous membranes are different from dermoscopic features of their counterparts located on the skin.

In the case of mucous membranes dermoscopy is significantly less investigated and less popular among clinicians. There is a need to intensify research, which would result in creating clear-cut dermoscopy criteria for pigmented mucosal lesions. This could be facilitated by creation of thin, flexible dermoscopes suitable for investigation of oral mucous membranes.

The lack of a widely available in-office technique generates the persistent risk of exposing patients to unnecessary excisional biopsies and extensive surgery, often resulting in significant disfigurement. A major part of these procedures would not be performed if oral or labial melanoma could be excluded beforehand by means of mucosal dermoscopy.

\section{References}

1. Esmaeili A 4th, Scope A, Halpern AC, Marghoob AA. Imaging techniques for the in vivo diagnosis of melanoma. Semin Cutan Med Surg. 2008; 27: 2-10.

2. Malvehy J, Puig S, Argenziano G, Marghoob AA, Soyer HP, International Dermoscopy Society Board members. Dermoscopy report: proposal for standardization. Results of a consensus meeting of the International Dermoscopy Society. J Am Acad Dermatol. 2007; 57:84-95.

3. Meleti M, Vescovi P, Mooi WJ, van der Waal I. Pigmented lesions of the oral mucosa and perioral tissues: a flow-chart for the diagnosis and some recommendations for the management. Oral Surg Oral Med Oral Pathol Oral Radiol Endod. 2008; 105: 606-616.

4. Buchner A, Merrell PW Carpenter WM. Relative frequency of solitary melanocytic lesions of the oral mucosa. J Oral Pathol Med. 2004; 33: 550-557.

5. Kahn MA, Weathers DR, Hoffman JG. Transformation of a benign oral pigmentation to primary oral melanoma. Oral Surg Oral Med Oral Pathol Oral Radiol Endod. 2005; 100: 454-9. 
6. Magremanne M, Vervaet C. Melanoma of the oral mucosa. Rev Stomatol Chir Maxillofac. 2008; 109: 175-177.

7. Hashemi Pour MS. Malignant melanoma of the oral cavity: a review of literature. Indian J Dent Res. 2008; 19: 47-51.

8. Kauzman A, Pavone M, Blanas N, Bradley G. Pigmented lesions of the oral cavity: review, differential diagnosis, and case presentations. J Can Dent Assoc. 2004; 70: 682-683.

9. De Giorgi V, Massi D, Carli P. Dermoscopy in the management of pigmented lesions of the oral cavity. Oral Oncol. 2003; 39: 534-535.

10. Hatch CL Pigmented lesions of the oral cavity. Dent Clin North Am. 2005; 49: 185-201.

11. Eisen D. Disorders in pigmentation in the oral cavity. Clin Dermatol. 2002; 18: 579-587.

12. Buchner A, Leider AS, Merrell PW, Carpenter WM. Melanocytic nevi of the oral mucosa: a clinicopathologic study of 130 cases from northern California. J Oral Pathol Med. 1990; 19: 197-201.

13. Puig S, Malvehy J. Dermoscopic findings of pigmented lesions of the mucosae. In Principles of Dermoscopy: Malvehy J, Puig S (Ed) Cage Editors. 2002; 289-299.

14. Meleti M, Leemans CR, Mooi WJ, Vescovi P, van der Waal I. Oral malignant melanoma: a review of the literature. Oral Oncol. 2007; 43: 116-121.

15. Jahanbani J, Forouzandeh A, Sadri D, Mirlashari J. Oral malignant melanoma diagnosed in an Iranian population over an 11-year period. Oral Maxillofac Surg. 2008 Aug 5. (epub ahead of print).

16. Owens JM, Gomez JA, Byers RM. Malignant melanoma in the palate of a 3-month-old child. Head Neck. 2002; 24: 9194.

17. Ariyoshi Y, Shimahara M, Omura K, Yamamoto E, Mizuki H, Chiba H, Imai Y, Fujita S, Shinohara M, Seto K. Epidemiological study of malignant tumors in the oral and maxillofacial region: survey of member institutions of the Japanese Society of Oral and Maxillofacial Surgeons, 2002. Int J Clin Oncol. 2008; 13: 220-228.

18. Hicks MJ, Flaitz CM. Oral mucosal melanoma: epidemiology and pathobiology. Oral Oncol. 2000; 36: 152-169.

19. González-García R, Naval-Gías L, Martos PL, Nam-Cha SH, Rodríguez-Campo FJ, Muñoz-Guerra MF, Sastre-Pérez J. Melanoma of the oral mucosa. Clinical cases and review of the literature. Med Oral Patol Oral Cir Bucal. 2005; 10: 264-271.

20. Hashemi Pour MS. Malignant melanoma of the oral cavity: a review of literature. Indian J Dent Res. 2008; 19: 47-51.

21. Disky A, Campos D, Benchikhi H. Case report: Mucosal melanoma of the lip and the cheek. Dermatol Online J. 2008; 14: 20.

22. Tanaka N, Mimura M, Kimijima Y, Amagasa T. Clinical investigation of amelanotic malignant melanoma in the oral region. J Oral Maxillofac Surg. 2004; 62: 933-937.
23. Matsushita S, Kageshita T, Ishihara T. Comparison of dermoscopic and histopathological findings in a mucous melanoma of the lip. Br J Dermatol. 2005; 152: 1324-1326.

24. Zampino MR, Borghi A, Corazza M, Virgili A. A preliminary evaluation of polyvinyl chloride film use in dermoscopic analysis of mucosal areas. Arch Dermatol. 2005; 141: 10441045.

25. Massi D, Nardini P, De Giorgi V, Carli P. Simultaneous occurrence of multiple melanoma in situ on sun-damaged skin (lentigo maligna), solar lentigo and labial melanosis: the value of dermoscopy in diagnosis. J Eur Acad Dermatol Venereol. 1999; 13: 193-197.

26. Stolz W, Braun-Falco O, Bilek P, Landthaler M, Burgforf W, Cognetta AB. Color Atlas of Dermatoscopy. Blackwell Publishing, 2002; 151-154.

27. Gaeta GM, Satriano RA, Baroni A (2002). Oral pigmented lesions. Clin Dermatol. 2002; 20: 286-288.

28. Hatch CL. Pigmented lesions of the oral cavity. Dent Clin North Am. 2005; 49: 185-201.

29. Kauzman A, Pavone M, Blanas N, Bradley G. Pigmented lesions of the oral cavity: review, differential diagnosis, and case presentations. J Can Dent Assoc. 2004; 70: 682-683.

30. Gencoglan G, Gerceker-Turk B, Kilinc-Karaarslan I, Akalin T, Ozdemir F. Dermoscopic findings in Laugier-Hunziker syndrome. Arch Dermatol. 2007; 143: 631-633.

31. Olszewska M., Banka A.: The usefulness of dermoscopy in monitoring pigmented oral lesions. Dermatologica. 2006; 6: 56-61.

32. Carli P, De Giorgi V, Cattaneo A, Giannotti B. Mucosal melanosis clinically mimicking malignant melanoma: non-invasive analysis by epiluminescence microscopy. Eur J Dermatol. 1995; 6: 434-436.

33. Mannone F, De Giorgi V, Cattaneo A, Massi D, De Magnis A, Carli P. Dermoscopic features of mucosal melanosis. Dermatol Surg. 2004; 30: 1118-1123.

34. Brooks JK, Nikitakis NG.Gingival pigmentation of recent origin. Oral melanoacanthoma. Gen Dent. 2008; 56:105.

35. Yarom N, Hirshberg A, Buchner A. Solitary and multifocal oral melanoacanthoma. Int J Dermatol. 2007; 46: 12321236.

36. Carlos-Bregni R, Contreras E, Netto AC, Mosqueda-Taylor A, Vargas PA, Jorge J, León JE, de Almeida OP. Oral melanoacanthoma and oral melanotic macule: a report of 8 cases, review of the literature, and immunohistochemical analysis. Med Oral Patol Oral Cir Bucal. 2007; 12: E374-379.

37. Rossiello L, Zalaudek I, Ferrara G, Docimo G, Giorgio CM, Argenziano G. Melanoacanthoma simulating pigmented spitz nevus: an unusual dermoscopy pitfall. Dermatol Surg. 2006; 32: 735-737. 